\title{
Management of Anticoagulant Therapy: The Dutch Experience
}

Frits R. Rosendaal, Felix J.M. van der Meer, and Suzanne C. Cannegieter

Anticoagulation Clinic Leiden, and Departments of Hematology and Clinical Epidemiology, University Hospital Leiden, The Netherlands

\begin{abstract}
In the Netherlands, outpatient oral anticoagulant therapy is monitored by a system of specialized anticoagulation clinics whose purpose is to make anticoagulant therapy as safe and effective as possible. In this paper we present the daily routine in one of these anticoagulation clinics (the Leiden clinic), which serves a catchment area with about $\mathbf{5 0 0 , 0 0 0}$ inhabitants. Several levels of quality control can be distinguished in the anticoagulation clinic. One level is the evaluation and standardization of laboratory methods, which includes participation in multicentered quality assurance programs. A second level is therapeutic quality control, which is the evaluation of how well a clinic maintains the patients within the specified target range of anticoagulant intensity. A third level is clinical quality control, which includes clinical outcome research. Outcome research includes studies into complications associated with treatment (e.g., bleeds), risk factors of treatment, and the optimal intensity of anticoagulant treatment.
\end{abstract}

Key Words. anticoagulant, quality control, management

Quality controi is at the core of anticoagulant therapy. It is the raison d'etre of the system of specialized anticoagulation clinics in the Netherlands [1]. Quality control starts with maintaining reliable and reproducible laboratory methods. It includes therapeutic quality control, which is the evaluation of how well patients are maintained at the desired intensity of anticoagulation. Clinical quality control is the evaluation of unwanted side effects of treatment (notably bleeds), and the evaluation of overall outcome of oral anticoagulant therapy, efficacy, and safety.

Quality control activities are motivated by a continuous effort to improve the care of patients. It includes participation in standardization programs and multicenter quality assurance programs to maintain the highest laboratory standards; efforts to extend the time spent by patients in the therapeutic range by ongoing training and feedback to all medical and paramedical staff involved in patient care; and maintenance of a sensitive system to record bleeds. Research is also a form of quality control. Research on the frequency of bleeds and the optimal intensity of oral anti- coagulant therapy in order to optimize the balance of therapeutic effect and side effects may be translated to improved quality of patient care.

\section{The Dutch System}

The Netherlands is a densely populated country at the North Sea with almost 16 million inhabitants. Seventy geographical regions are served by anticoagulation clinics [2]. Every patient who needs anticoagulant therapy is referred to the clinic in his or her region for anticoagulant monitoring. Since reimbursement is based on participation in this system of specialized anticoagulation clinics, very few, if any, patients receive outpatient treatment with coumarin outside this system.

Taken as a group, the anticoagulation clinics form the Federation of Dutch Thrombosis Services, which is an organization that sets guidelines for treatment, facilitates laboratory quality control, organizes conferences and postgraduate training, promotes research, and publishes its own newsletter. In addition to its Dutch members, the federation includes two anticoagulation clinics that are located in Spain, and provides service in areas where many Dutch elderly live in the winter.

Two types of coumarin derivatives are licensed for oral anticoagulant therapy in the Netherlands: shortacting acenocoumarol (Sintrom mitis ${ }^{\circledR}$ ) and long-acting phenprocoumon (Marcoumar $\left.{ }^{\circledR}\right)$. The federation has established three target INRs for the intensity of anticoagulation: INR of 3.0 for venous thromboprophylaxis, INR of 3.5 for arterial thromboprophylaxis, and INR of 4.0 for prophylaxis of thromboemboli in patients with mechanical heart valves (Table 1). Overall figures on quality control collected by the federation show that in 1 year, up to $65 \%$ of INRs are within these target ranges, about $25 \%$ are below, and about $10 \%$ are over the desired range.

Address for correspondence: Dr. F.R. Rosendaal, Department of Clinical Epidemiology, Building 1, C0-P, University Hospital Leiden, P.O. Box 9600, 2300 RC Leiden, The Netherlands. 
Table 1. Indications and target intensities for oral anticoagulant therapy Federation of Dutch Thrombosis Servees recommendations

\begin{tabular}{ll}
\hline INR & Indication \\
\hline $25-35$ & $\begin{array}{c}\text { Prophylaxis of venous thrombosis after surgical in- } \\
\text { terventions, during immobilization, after recur- } \\
\text { rent venous thrombosis } \\
\text { Treatment of venous thrombosis in acute deep vem } \\
\text { thrombosis, in acute pulmonary embolism } \\
\text { Prophylaxis of arterial thrombosis after myocardial } \\
\text { infarction, after ischemic stroke, in atrial fibrilla- } \\
\text { tion, in cardiomyopathy, after coronary bypass or } \\
\text { PTCA, in angina pectoris, in peripheral arterial } \\
\text { disease, in heart valve disease } \\
\text { Prosthetic heart valves }\end{array}$ \\
\hline
\end{tabular}

PTCA $=$ percutaneous transluminal coronary angioplasty

\section{Leiden Anticoagulation Clinic}

The anticoagulation clinic for the city of Leiden serves as an example of a Dutch clinic. It takes care of the monitoring of outpatient anticoagulant therapy for patients in the region of Leiden. This region includes the city of Leiden as well as a rural area and serves, in total, an area with about 500,000 inhabitants. Approximately 7000 patients are treated annually by the Leiden Anticoagulation clinic ( 1 in every 70 inhabitants of the region receive short- or long-term treatment with coumarin in a given year). The Leiden Anticoagulation Clinic is closely associated with the Department of Hematology, Section of Hemostasis and Thrombosis of the University Hospital Leiden, and also monitors anticoagulation with coumarm and heparm in inpatients of the hospital. (Because a Dutch anticoagulation clinic does not usually monitor inpatient care, we will not discuss it further here.)

Approximately 500 patients are seen daily by a nurse of the anticoagulation cline at the clinic, at small facilities throughout the region, or at the home of bedridden patients. The nurse, who is specialized in anticoagulant care, has a central role $\mathrm{m}$ the system. She sees the patients personally and takes a small standardized medical history, with regard to bleeds, other diseases, and change of medication. The nurse educates the patient, especially about when to contact the anticoagulation clinic between visits. Finally, the nurse has a central role in communicating patient information back to the physician.

Prothrombin times are measured at the anticoagulation clinic from the specimens brought by the nurse; a bar-code reader enters patient identification into the computer system; the prothrombin time, converted into an INR, is directly transferred to the computer system; and if necessary, the nurse adds specific information from the medical interview.

The dosage and time interval to the next checkup are decided by specialized physicians, assisted by a computer algorithm that suggests a dosage and time period for stabilized patients. The physicians either authorize the suggested dosage or enter a specific dosage directly into the computer. The physicians specify a mean daily dosage, which is translated into the specific dose for each day by the algorithm.

After dosages and time intervals are determined for all patients, the computer prints a calendar for each patient, indicating how many tablets to take each day. These calendars are sent by mail and arrive the next morning. On these calendars a bar-code label is printed, which can be peeled off, and is stuck to the Vacutainer at the next blood draw. In case immediate action is required, for example, in extremely overanticoagulated patients, patients are contacted by phone. The time period between visits varies from 1 to 6 weeks, with an average slightly over 3 weeks.

The Leiden anticoagulation system has been computerized since 1973 [3]. This system generates a dose prescription in 55\% for all prothrombin times measured. It further helps to organize the appointments for visits or house calls by the nurses, and to generate daily overviews for quality control, for example, of patients for whom the dosage was changed by more than $15 \%$ and of the number of INRs within the therapeutic range.

\section{Therapeutic Quality Control}

One of the most important aims for anticoagulant therapy monitoring is to maintain prothrombin times within the target zone. There are several ways to express the success of this effort [4-6]. A simple proportion of INRs within the therapeutic range gives an unduly pessimistic result because patients who are unstable are seen most often [7].

We recently proposed a method that assumes a linear change of the INR between visits and counts the number of days spent by all patients at different INRs, similar to the categorized person-time methods used to calculate incidence rates [8]. This method can be used for two distinct purposes: (1) to express the therapeutic quality of anticoagulation as the proportion of the total observation time of all patients that was spent in the target zone; (2) to relate the persontime at various INRs to the INRs observed at adverse events, and from that to calculate the risk of these events at different intensities of anticoagulation. We have used this method in several studies, two of which are described here.

\section{Clinical Quality Control}

In 1 year, a total of almost 7000 patients were treated with oral anticoagulants at the Leiden anticoagulation clinic, most with long-term treatment. These patients had a mean age of 66 years, with all ages represented, from the young to the very old. About two thirds of 
all patients were men, with the predominance of men completely explained by a higher prevalence of arterial disease among men (Table 2).

Much of the efforts in an anticoagulation clinic are aimed at the reduction of side effects, especially bleeds. We analyzed the experience of our patients over a 1-year period, to assess which factors contributed to the risk of bleeding [9]. We classified all bleeds that were fatal, intracranial, or that required hospital admission, tranfusions, or surgical interventions, as well as all bleeds in muscles and joints, as major bleeding.

The overall frequency of major bleeds was $2.7 \mathrm{per}$ 100 patient-years, that is, $2.7 \%$ per year $(95 \%$ CI 2.3-3.1\%). The incidence of intracranial bleeding was 0.62 per 100 patient-years, and the incidence of fatal bleeding was 0.64 per 100 patient-years. Over half of all bleeding-related deaths were caused by hemorrhagic strokes. These bleeding rates are similar to those found in other studies, although there have been reports of higher [10] as well as lower rates [11]. Much of the discrepancy appears to be related to patient selection criteria. Selection of complying patients with limited or no co-morbidity for a randomized trial may reduce the observed incidence of bleeds. An incidence of $2-4 \%$ per year probably reflects what can be reached in a clinic dedicated to anticoagulation therapy in unselected patients.

Can this risk of bleeding be reduced further? One approach to this question is to assess which patients are most at risk of bleeds. In a multivariate regression model, we looked at several factors that might influence risk. We found a slight $16 \%$ excess of bleeding in women as compared with men, with a very wide confidence interval (95\% CI 0.83-1.61, for the relative risk). The strongest predictors of the bleeding risk were age and INR, that is, the achieved intensity of oral anticoagulation as calculated by the method we described previously.

For each 10-year increase in age, the risk of bleeding increased $46 \%$, and for each unit increase in INR, the frequency of bleeds rose by $42 \%$. In absolute figures, the risk of major bleeds was still less than $2 \%$ in those aged $45-64$, but rose to over $5 \%$ in those aged 75 and older. These findings imply that older individuals with a higher INR will have a severalfold increase in risk compared with that of younger individuals with a lower INR.

In this study, we looked at bleeding complications only, not at the prevention of thromboembolic events. Therefore, we cannot derive recommendations from it regarding the intensity of anticoagulation, and specifically cannot recommend the use of lower INRs for older patients, since we have no information on the rate of thromboembolization in relation to age and INR. It does follow from these results, however, that it is especially worthwhile to attempt to avoid overanticoagulation in the elderly, which may be achieved by more frequent monitoring.

\section{Optimal Intensity of Oral Anticoagulation}

When a treatment depends upon such a fine balance of therapeutic effect and side effect, finding the right dosage becomes crucial. Anticoagulation clinics with dedicated physicians and nurses are designed to maintain this balance and to maintain the patients on the desired intensity of anticoagulation as long as possible. Research is needed to find which intensity is optimal, that is, which will offer the lowest risk of thromboembolic and bleeding complications. This research is hampered by the variation of INRs over time. A randomized clinical trial, in which two target INRs or target ranges are compared, will therefore not determine which intensity is optimal: It will compare two strategies, which will not have been maintained all of the time in all of these patients due to the normal variation of the INR.

We reasoned that the optimal intensity would be the intensity at which the frequency of complications was lowest, supposing that we were able to maintain that intensity all of the time. Therefore, we calculated the incidence of complications over the whole range of achieved INRs, by the method mentioned earlier [8]. With this method, for each intensity the incidence of events is calculated as the number of events occurring at that INR divided by the total person-time spent at that INR. This is the standard way to calculate incidence rates, with the main difference being

Table 2. Characteristics of the patients at the Leiden Anticoagulation Clinic

\begin{tabular}{|c|c|c|c|c|c|}
\hline & \multicolumn{2}{|c|}{ Men } & \multicolumn{2}{|c|}{ Women } & \multirow[b]{2}{*}{ Overall } \\
\hline & Arterial disease & Other indications & Arterial disease & Other indications & \\
\hline Number of patients & 3511 & 1106 & 1103 & 1094 & 6814 \\
\hline Patient years & 3268 & 926 & 1004 & 887 & 6085 \\
\hline Major bleeds (n) & 66 & 35 & 26 & 35 & 162 \\
\hline Major bleeds (\%/yr) & 2.0 & 3.8 & 2.6 & 4.0 & 2.7 \\
\hline Fatal bleeds (n) & 12 & 14 & 4 & 10 & 39 \\
\hline Fatal bleeds (\%/yr) & 0.4 & 1.5 & .06 & 0.8 & 0.6 \\
\hline
\end{tabular}


that the person-time at each INR 1s contributed to by different patients.

A problem, in addition to this distribution of the person-time of each patient over the various intensities, is to estimate the INR in each patient on each individual day. Obviously, this is not known, but a reasonable estimate is a linear interpolation between successive visits. This way of deriving "INR-days" is depicted in Figure 1. The person-time of the full cohort is distributed in INR-specific person-time in this way. Subsequently, for all patients with events, the INR at the time of the event is obtained from patient records or hospital charts. For each INR, the incidence of events at that intensity is then calculated as all events occurring at that INR divided by the total person-time at that INR.

The incidence rates calculated in this fashion are our best estimate of the frequency of untoward events at different intensities of anticoagulation, if one were able to maintain patients at each of these intensities permanently. This may be viewed as the "ideal pharmacological" effect of anticoagulation, which may subsequently serve to set target INRs and target ranges, and to design studies aimed at improving the stability of anticoagulation therapy.

\section{Leiden Artificial Valves and Anticoagulation Study (LAVA)}

Patients with artificial heart valves are at high risk of valve thrombosis and embolization. Therefore, they receive the most intense anticoagulant therapy, and are also most at risk of bleeding [12]. Because striking the proper balance between thromboemboli and bleeding is most critical in this patient group, we set out to assess the optimal intensity of anticoagulation [13].

We followed 1608 patients from four anticoagulation clinics over an average 4 years and registered all INR measurements over this period and all major complications, bleeds, and thromboemboli. We calculated the incidence of these untoward events for each intensity of anticoagulation.

Over 6475 years of follow-up, we registered 210 (3.5 per 100 patient-years) major events, 45 thromboemboli ( 0.7 per 100 patient-years), and 164 major bleeds (2.7 per 100 patient-years). Strokes occurred in 93 patients (1.4 per 100 patient-years), and fatal bleeding in 20 patients $(0.30$ per 100 patient-years).

The incidence of untoward events was strongly dependent on the intensity of anticoagulation (Figure 2). Whereas the overall incidence was $3.5 \%$ per year, it was $2 \%$ when INRs between 2.5 and 4.9 were achieved. It increased sharply with lower INRs to 7.5\% per year for INR 2.0-2.5 up to $27 \%$ per year for achieved INRs between 1.0 and 1.4. This increased risk in under-anticoagulated patients was due to increasing rates of embolism; when INRs higher than 4.9 were achieved, the frequency of bleeding rose

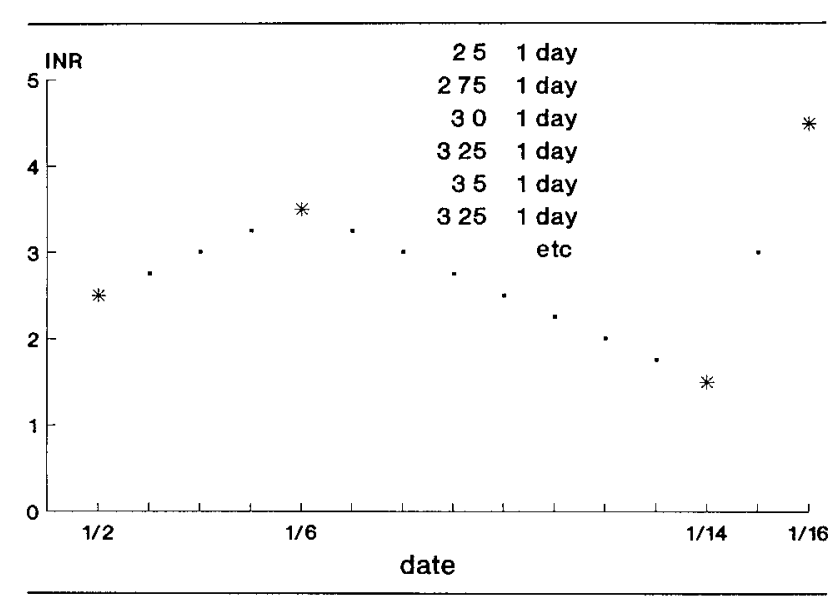

Fig. 1. Method to calculate INR-specific incudence rates The figure represents one patzent, who has been seen four times between January 2 and 16 (marked by asterisks), with actual $I N R$ values of $25,35,10$, and 45 measured at those visits When a linear change of the INR between visits is assumed, achreved INR can be assigned for each day 25 at 1/2, 275 at 113,30 at 1/3, and so on The days at each INR will be summed, per patzent (so this one patzent contmbutes 3 days to INR 30 , at 1/4, at 1/8, and at 1/15) and then over all of the patients Thus yzelds a total person time at each INR, which is the denominator for the event rate at each INR The numerator is the number of events at each INR, for all patients with events, as denved from the patient records

sharply: 4.8 per 100 treatment-years for INR $5.0-5.5$, up to 75 per 100 patient-years at an INR over 6.5 .

When we looked only at the classified strokes, the risk of ischemic stroke went up when the INR was lower, while for hemorrhagic strokes the risk went up with increasing intensities of anticoagulation. The high risk of embolization in under-anticoagulated patients, and the high risk of bleeding in overanticoagulated patients, is reflected in the $\mathrm{U}$-shaped curve shown in Figure 2. When we limited the analysis to the most severe adverse effects (i.e., only those that were fatal or led to a lasting handicap with dependency), again the optimal intensity was found between INRs of 2.5 and 4.9 .

These data indicate that when a constant INR was achievable, the optimal level would lie between 2.5 and $4.9 \mathrm{INR}$, at which the rate of complications would be almost halved compared with the current frequency of untoward events. Within this range, the risks did not vary much by INR. Since achieved INRs will vary within and around target INR ranges, from these data it follows that a reasonable target range lies between 3.0 and $4.0 \mathrm{INR}$.

\section{Conclusions}

The system of specialized anticoagulation clinics in the Netherlands has been designed to optimize the treatment of patients who require oral anticoagulants. This 


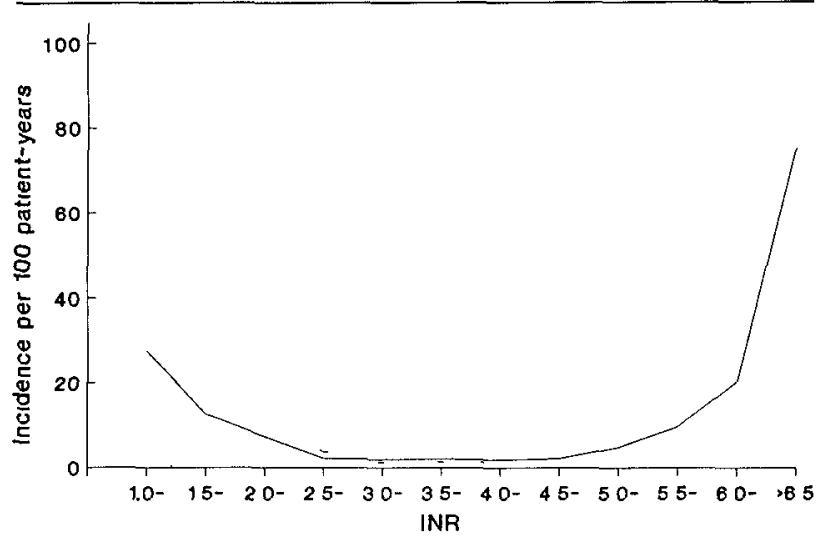

Fig. 2. Incidence of all adverse events (all thromboembolt, major bleeds, and strokes) by intensity of anticoagulation INRspecific incidence rates for all major adverse events per 100 person-years. The dotted lines are the $95 \%$ confidence intervals around the incidence (Reprinted by permission of The New England Journal of Medicine, Cannegneter SC, Rosendaal FR, Wintzen AR et al., 1995;383:11-1\%. Copyrght 1995. Massachusetts Medical Soczety. All rights reserved)

treatment will benefit from ongoing quality control, not only of the laboratory methods, but also of the performance of the clinic in maintaining the patients within a target range. Finally, the concentration of patients allows for studies designed to further improve care by investigating risk factors for complications and by assessing the safest intensity of anticoagulation.

\section{References}

1. Loeliger EA, van Dijk-Wierda CA, van den Besselaar AMHP, Broekmans AW, Roos J. Anticoagulant control and the risk of bleeding. In: Meade TW, ed. Anticoagulants and Myocardial Infarction A Reappraisal New York: John Wiley, 1984:135-177.

2. Van den Besselaar AMHP, Van der Meer FJM, GerritsDrabbe CW. Therapeutic control of oral anticoagulant treatment in the Netherlands. Am J Clin Pathol 1988;90: $685-690$.

3. Wiegman H, Vossepoel AM. A computer program for long term anticoagulant control. Comput Methods Programs Bromed 1977;7:71-84.

4. Copplestone A, Roath S. Assessment of therapeutic control of anticoagulation. Acta Haematol 1984;71:376-380.

5. Duxbury BM. Therapeutic quality control leading to further clinical assessment of oral anticoagulation. Acta Haematol 1986;76:65-67.

6. Van den Besselaar AMHP. Recommended method for reporting therapeutic control of oral anticoagulant therapy. Thromb Haemost 1990;63:316-317.

7. Duxbury BM. Therapeutic control of anticoagulant treatment. Br Med $J$ 1982;284:1634-1635.

8. Rosendaal FR, Cannegieter SC, Van de Meer FJM, Briet $\mathrm{E}$. A method to determine the optimal intensity of oral anticoagulant therapy. Thromb Haemost 1993;69:236-239.

9. van der Meer FJM, Rosendaal FR, Vandenbroucke JP. Bleeding complications in oral anticoagulant therapy: An analysis of risk factors. Arch Intern Med 1993;153: $1557-1562$.

10. Landefeld CS, Goldman L. Major bleeding in outpatients treated with warfarin: Incidence and prediction by factors known at the start of outpatient therapy. AmJ Med 1989,87: 144-152.

11. Smith P, Arnesen H, Holme I. The effect of warfarin on mortality and reinfarction after myocardial infarction. $N$ Engl J Med 1990;323:147-152.

12. Cannegieter SC, Rosendaal FR, Briet E. Thromboembolic and bleeding complications in patients with mechanical heart valve prostheses. Crrculation 1994;89:635-641.

13. Cannegieter SC, Rosendaal FR, Wintzen AR, Van der Meer FJM, Vandenbrouck JP, Briet E. Optimal oral anticoagulation therapy in patients with mechanical heart valves. $N$ Engl J Med 1995;333:11-17. 